\title{
Criminologie
}

\section{Billets des directeurs de Criminologie}

Volume 41, numéro 1, printemps 2008

40 ans de Criminologie : perspectives d'avenir

URI : https://id.erudit.org/iderudit/018417ar

DOI : https://doi.org/10.7202/018417ar

Aller au sommaire du numéro

Éditeur(s)

Les Presses de l'Université de Montréal

ISSN

0316-0041 (imprimé)

1492-1367 (numérique)

Découvrir la revue

Citer cet article

(2008). Billets des directeurs de Criminologie. Criminologie, 41(1), 9-16.

https://doi.org/10.7202/018417ar d'utilisation que vous pouvez consulter en ligne.

https://apropos.erudit.org/fr/usagers/politique-dutilisation/ 


\title{
Billets des directeurs de Criminologie
}

\section{Les 40 ans de Criminologie: une étape importante dans l'institutionnalisation de la discipline}

\author{
Denis Szabo ${ }^{1}$
}

Fondateur de Criminologie, directeur de 1968 à 1987

Lorsque fut créé en 1960 le programme de Maîtrise ès Arts en Criminologie à l'Université de Montréal, ma préoccupation principale était d'assurer à long terme le prestige scientifique de la nouvelle discipline. En effet, les enseignements déjà bien établis tels que la psychologie et les autres sciences sociales considéraient avec un certain scepticisme la vocation scientifique de notre discipline. Celle-ci, dès l'abord, était d'orientation professionnelle. Ainsi, le service social, déjà bien établi, fut reconnu par sa valeur de formation pratique; beaucoup cependant s'interrogeaient sur sa valeur proprement scientifique.

Le danger pour la criminologie était évident: nos diplômés étaient en effet destinés, essentiellement, à l'intervention auprès des délinquants, des déviants, des détenus, des personnes judiciarisées et à la gestion des services d'administration de la justice et de la sécurité publique. Or, cette intervention devait avoir, à l'instar de la médecine, un fondement scientifique si elle devait se qualifier pleinement comme une véritable discipline d'enseignement. La vocation vers la pratique de l'intervention était incontournable: c'était la justification même de la formation de nos criminologues. Mais pour en assurer la qualité basée sur la recherche scientifique, il était indispensable que le nouveau département et par la suite École soit aussi un centre de recherche de haut niveau.

1. Professeur émérite, École de criminologie, Université de Montréal. 
C'est là que se trouvait le défi que l'équipe toute jeune du département devait relever. Or, un important critère de la qualité de cette recherche était la publication des résultats de nos travaux. Ceux-ci devaient être présentés à l'attention critique du monde scientifique national et international. La qualité particulière, originale, de caractère interdisciplinaire de l'équipe des enseignants/chercheurs méritait l'attention du monde académique: médecins, psychologues, sociologues, juristes, politologues conjuguaient leurs efforts dans l'étude des sources, des formes, des caractéristiques des comportements délinquants et de la réaction sociale que ceux-ci provoquent.

C'est cette préoccupation de présenter la criminologie comme une science qui a présidé en 1968 au choix du premier titre de la revue, Acta criminologica, qui soulignait le sérieux de sa vocation scientifique. Les premiers numéros contenaient de substantiels mémoires produits par les chercheurs de l'équipe, écartant toute référence à l'actualité de la scène criminelle. Au fil des années, plus confiants dans nos forces, nous passions en 1975 à la formule, qui existe jusqu'à nos jours, des numéros thématiques. Les thèmes les plus divers embrassent carrément l'actualité que présente la politique criminelle en pleine transformation, engageant des réformes majeures de modernisation de nos appareils institutionnels. L'examen des titres témoigne bien de ce souci de service et reflète les divers aspects de l'actualité de la question criminelle au Québec et au Canada. La revue prend ainsi sa place parmi les publications périodiques bien établies dans le monde national et international.

Ainsi fut assurée l'intégration harmonieuse autour du socle que représente l'Université (École de criminologie, Centre international de criminologie comparée [CICC]) d'une part, et la pratique professionnelle d'autre part. La revue devenait le véhicule même de l'information et de la formation permanente de nos criminologues. Ceux-ci comptent actuellement environ 2500 membres diplômés, engagés dans des secteurs de plus en plus nombreux et étendus de l'administration de la justice pénale, qu'il s'agisse de la dissuasion, de la prévention de la délinquance aussi bien que de l'administration des peines, du traitement et de la resocialisation des auteurs d'actes criminels.

Criminologie fut ainsi établie sur des fondations solidement ancrées dans une réalité toujours mouvante. Son programme de publications a été inspiré d'une pensée stratégique d'innovation et d'adaptations permanentes. Cela résultait d'une interaction dynamique entre l'université et le monde de plus en plus diversifié des pratiques professionnelles. 
Grâce à cette institutionnalisation, les criminologues montréalais sont en mesure de continuer à jouer un rôle important dans le monde de la criminologie tant nationale qu'internationale.

Grâce au travail et au dévouement de plusieurs générations d'équipes de direction: Szabo-Parizeau (1968-1987), Normandeau-De Plaen (1987-1989), Brochu-De Plaen (1989-1997), Landreville-Gravel/ Arcand (1997-2005), Casoni-Pacheco (2005...), notre revue sera présente lors de la commémoration du demi-siècle de l'institutionnalisation de la criminologie à l'Université de Montréal en l'an 2010.

\section{Criminologie dans les années 1980}

\section{André Normandeau ${ }^{2}$}

Directeur de 1987 à 1989

À titre de directeur de la revue Criminologie au cours des années 1980, j'ai eu le plaisir de relever deux ou trois défis intéressants liés à la politique rédactionnelle de la revue, à savoir:

1. Je succédais à ce titre à un «monstre sacré», soit le Dr. Denis Szabo, le fondateur de la revue en 1968, mais également le directeur-fondateur de l'École de criminologie de l'Université de Montréal (1960-1970) et le directeur-fondateur du Centre international de criminologie comparée de l'Université de Montréal (1970-1983). Denis Szabo avait créé une revue de portée internationale, du moins dans le réseau criminologique des pays entièrement et partiellement francophones. À la demande de mes collègues de l'époque, mon premier défi fut d'inscrire notre revue de façon plus sensible dans le réseau national québécois. Dans cette perspective, je fus amené à choisir des thèmes plus près des intérêts des chercheurs et des praticiens du Québec et d'obtenir plus de collaboration de ces derniers dans la rédaction des différents numéros de la revue.

2. À l'équilibre «international-national» s'ajouta également un équilibre entre les auteurs issus de la recherche universitaire «et» les auteurs qui travaillaient au niveau de la pratique quotidienne: sécurité publique et sécurité privée; tribunal (adulte et juvénile); services correctionnels:

2. Professeur à la retraite, École de criminologie, Université de Montréal. Professeur, Université de Toulouse. 
probation, prison, libération conditionnelle...; services d'aide aux victimes, aux toxicomanes, aux ex-criminels en quête d'emploi, aux jeunes en difficulté... Cet équilibre «théorie/recherche-pratique» fut bien reçu, par le réseau des praticiens en particulier, puisque l'Association professionnelle des criminologues du Québec (APCQ) et la Société de Criminologie du Québec (SCQ) décidèrent alors d'abonner «automatiquement» leurs membres à notre revue.

3. «À tout seigneur, tout honneur!» Il est important de souligner ici le rôle exemplaire, dans la recherche des deux équilibres mentionnés, de madame Alice (Ponanska) Parizeau. Secrétaire générale à l'époque du Centre international de criminologie comparée, elle participait pleinement à toutes les réunions du Comité de rédaction de la revue. Elle intervenait fréquemment pour nous rappeler l'importance de ces équilibres. D'origine polonaise, jeune réfugiée en France de la Seconde Guerre mondiale, diplômée en droit de l'Université de Paris, mariée a un futur premier ministre du Québec (Jacques Parizeau), carrière en journalisme au Québec, auteure d'une douzaine d'essais et de romans, femme engagée dans de multiples causes humanitaires liées aux droits des enfants maltraités, Alice Parizeau avait rédigé au début des années 1970 les deux volumes fort importants sur la justice des mineurs publiés par la Commission d'enquête sur l'administration de la justice au Québec, dite Commission Prévost (1970). Elle travailla par la suite au Centre international de criminologie comparée (1972-1987). Elle devait mourir du cancer en 1990.

4. Tout compte fait, les années 1980 ont été particulièrement importantes pour placer la revue Criminologie au cœur des préoccupations des criminologues québécois, tout en maintenant un niveau de qualité national et international qui ont fait de notre revue un outil reconnu dans les cercles criminologiques du Québec, soit, mais également en France, en Belgique, en Suisse et dans les pays africains francophones. En langue française, notre revue est certainement l'une des deux ou trois principales revues criminologiques.

Parole d'un ex-directeur ! 


\section{Les défis des années 1990}

\section{Serge Brochu ${ }^{3}$}

Directeur de 1989 à 1997

Le grand défi pour le directeur de Criminologie à la fin des années 1980 et au cours des années 1990 a été de réussir à gérer différentes crises, toutes extérieures à la revue, mais qui toutes ont eu une incidence directe sur nous. La première de ces «crises» a été celle de l'institutionnalisation du Fonds pour la formation des chercheurs et l'aide à la recherche (FCAR), qui avait été mis sur pied en 1984. Je suis en effet arrivé à la direction de la revue au moment où le FCAR révisait en profondeur ses critères de subvention en insistant d'une part sur les facteurs garantissant la neutralité du processus d'évaluation des articles et la transparence des processus décisionnels tout en exigeant, d'autre part, de plus grandes garanties de santé financière. Cela tombait au pire moment pour Criminologie, car nous étions, en même temps, et bien malgré nous, plongés dans une crise de production, les Presses de l'Université de Montréal (PUM) connaissant des difficultés importantes. Il nous était donc bien difficile de convaincre le FCAR au sujet de nos budgets, ne sachant trop bien nous-mêmes quels étaient nos réels coûts de production. À cela, s'est ajoutée la crise d'une baisse draconienne des abonnements qui résulta de la fin abrupte des activités de l'Association professionnelle des criminologues du Québec (APCQ), qui avait jusqu'alors généré une bonne partie de nos abonnements puisque chaque membre recevait un abonnement à Criminologie en se joignant à l'Association.

Avec l'aide inestimable de Jacqueline De Plaen, qui agissait comme secrétaire de rédaction et dont les connaissances en édition nous ont été très précieuses, ainsi que des membres du Conseil d'administration, la revue a fini par tirer son épingle du jeu. D’abord, en établissant des règles claires de fonctionnement tant sur le plan de ses divers comités que sur le plan des évaluateurs. Ensuite, en lançant une vaste campagne d'abonnements qui nous a permis d'aller chercher de nouveaux abonnés à la grandeur du Québec et même en Europe. La revue a aussi favorisé une collaboration fructueuse entre l'Association des étudiants de criminologie de l'Université de Montréal (AECUM), l'École de criminologie

3. Vice-doyen aux ressources humaines et à la gestion, Faculté des arts et des sciences. Professeur, École de criminologie, Université de Montréal. 
et le Centre international de criminologie comparée (CICC) afin qu'ensemble ils offrent un abonnement d'un an aux étudiants de maitrise de l'École de criminologie. Cette collaboration dure encore aujourd'hui, répondant à un besoin réel d'approfondissement des connaissances chez les étudiants et de soutien à la revue pour le CICC et l'École de criminologie. Enfin, en usant d'imagination, en sollicitant la collaboration des uns et des autres et en travaillant très fort, nous avons relevé ces divers défis; notamment en réussissant à convaincre le FCAR de continuer à nous subventionner, ce qui, vu d'aujourd'hui, a été une excellente affaire pour la recherche, comme pour le transfert des connaissances.

\section{Criminologie dans l'aire du numérique}

Pierre Landreville ${ }^{4}$

Directeur de 1997 à 2005

Je suis très heureux de m'associer à la revue Criminologie à l'occasion du $40^{\mathrm{e}}$ anniversaire de sa fondation par Denis Szabo.

J'ai été directeur de la revue de 1997 à 2005. Ce fut, dans une certaine mesure, un moment charnière dans son évolution. Nous avons eu, mes collègues du Conseil d'administration et moi, à engager cette dernière dans l'aire du numérique. Grâce au financement du Fonds québécois de la recherche sur la société et la culture (FQRSC) et des pionniers du portail Érudit, la revue est maintenant publiée en version papier et en version numérique. Un financement spécial a même permis d'en faire numériser toute la collection depuis le volume 1 de 1968. C'est un outil inestimable pour les chercheurs et un moyen extraordinaire de faire connaître la criminologie d'ici au monde entier. Nous sommes fiers de cette réalisation.

Par ailleurs, nous nous étions fixé deux autres objectifs en ce qui concerne le contenu de la revue. D'une part, nous avons cru que, tout en conservant l'accent sur la publication de recherches et de réflexions des chercheurs canadiens et québécois, il était souhaitable d'augmenter la contribution de chercheurs étrangers. Dans tous les numéros, nous avons sollicité des experts internationaux pour compléter les façons de voir sur un thème donné.

4. Professeur émérite, École de criminologie, Université de Montréal. 
D'autre part, nous avons été très vigilants pour maintenir l'ouverture de la revue à tous les points de vue portant sur la question criminelle. Comme l'avait souhaité Denis Szabo, fondateur de la revue, nous avons toujours insisté pour que Criminologie soit vraiment un carrefour interdisciplinaire ouvert à toutes les perspectives disciplinaires et théoriques et abordant tous les thèmes criminologiques que ce soient le passage à l'acte, la réaction sociale et pénale aux comportements criminalisés, le fonctionnement du système pénal ou les politiques criminelles. Des thèmes tels que l'approche développementale en prévention, la justice réparatrice, la justice des mineurs, la victimologie, la police, la cybercriminalité ou le génocide, qui ont été traités durant mon mandat, en témoignent.

Je crois fermement que la revue doit conserver cette ouverture et demeurer un lieu d'échanges de divers points de vue sur la question criminelle. Je souhaite longue vie à Criminologie en étant assuré qu'elle demeurera un moyen de diffusion et de confrontation de calibre international pour les chercheurs canadiens et québécois.

\section{Criminologie aujourd'hui}

\section{Dianne Casoni ${ }^{5}$}

Directrice depuis 2005

Les billets de mes prédécesseurs témoignent des défis auxquels la revue a dû se mesurer pour réussir à faire sa niche parmi les revues scientifiques tout en poursuivant sa mission d'offrir aux praticiens de la criminologie des connaissances récentes et pertinentes issues de la recherche. Ce désir de relever toujours plus la qualité scientifique de notre revue a récemment été grandement récompensé. Criminologie vient en effet de recevoir l'insigne honneur de se voir choisie par Ellen Cohn et David Farrington $^{6}$, dans leur plus récente analyse bibliométrique, parmi les 40 revues scientifiques - et seule revue non anglophone - sondées afin d'établir la liste des auteurs les plus cités dans le domaine. Criminologie reçoit ainsi un témoignage des plus éloquents attestant du fait que la revue a atteint son objectif premier qui est de publier une revue d'une

5. Professeure titulaire, École de criminologie, Université de Montréal.

6. Cohn, E. G., \& Farrington, D. P. (2008). Scholarly influence in criminology and criminal justice journals. 1990-2000. Journal of Criminal Justice, 36: 11-21. 
grande qualité et d'une indéniable crédibilité scientifiques. Notre inscription parmi les 40 revues qui comptent dans le monde scientifique de la criminologie en dit beaucoup sur la qualité du travail de nos nombreux collaborateurs, des membres des différents comités qui ont pour tâche de penser aux thèmes et de veiller à la qualité scientifique de la revue, aux responsables de numéros et aux auteurs des articles publiés.

Outre les collaborateurs de première ligne, membres des divers comités, responsables de numéros et auteurs, la revue n'aurait pu si bien se démarquer sans la précieuse collaboration de notre assistante à la rédaction, Chloé Leclerc, et de notre rédactrice en chef, Adriana Pacheco, toutes deux doctorantes en criminologie. Lappui fidèle que nous recevons de nos partenaires, l'École de criminologie et le CICC, qui se sont engagés dès la première heure à soutenir la revue mérite aussi largement d'être souligné. Il m'importe également de remercier les Presses de l'Université de Montréal qui nous publient, Fides qui distribue la revue et le consortium Érudit qui diffuse la version électronique de Criminologie. Chacun de ces partenaires a à cœur la réussite de notre revue et aucun ne ménage ses peines pour y parvenir, à preuve la récente course contre la montre des PUM afin de sortir le dernier numéro, Peines et pénalité au Canada. Autour des travaux de Pierre Landreville, à temps pour la tenue du colloque Le pénal aujourd'bui: pérennité ou mutations tenu à Montréal du 5 au 7 décembre 2007. Enfin, il faut rappeler que sans le soutien financier du CRSH et du FQRSC, nous ne fêterions pas aujourd'hui un si heureux anniversaire. Il me reste à vous convier tous à l'automne 2008 au colloque organisé pour fêter nos 40 ans; venez en grand nombre souligner notre succès à tous. 